\title{
Coronary artery calcification: does it predict obstructive coronary artery disease?
}

\author{
A. S. Dharampal • P. J. de Feyter
}

Published online: 12 July 2013

(C) The Author(s) 2013. This article is published with open access at Springerlink.com

Calcification of the coronary arteries plays a key role in the pathophysiology of atherosclerosis. Coronary calcification is an active process culminating in extracellular matrix deposition of calcium by osteoblast-like cells that has some resemblance to bone formation [1]. Subintimal coronary calcification is almost exclusively associated with the presence of coronary atherosclerosis and is considered the hallmark of coronary atherosclerosis. Coronary calcific lesions are considered advanced lesions and calcification following plaque rupture of a high-risk plaque is thought to be part of the healing process [2].

The presence of coronary calcification is a surrogate marker of the overall plaque burden. Not all plaques are calcified and histological studies have revealed that the total calcium area was approximately $20 \%$ of the total atherosclerotic plaque burden area [3].

There is no generally accepted relationship between plaque calcification and plaque stability. Traditionally calcific plaques are considered to be stable but plaques with spotty calcification (small amount of calcification in a spotty distribution) have been associated with plaque instability and acceleration to progression of $\mathrm{CAD}[4,5]$. However, the extent of calcification is a marker of instability somewhere in the coronary tree rather than a marker of local plaque instability [4].

\section{A. S. Dharampal $(\bowtie) \cdot$ P. J. de Feyter}

Department of Radiology, Erasmus MC, Room Ca 207a,

's-Gravendijkwal 230, 3015 CE, Rotterdam, the Netherlands

e-mail: anoesh_dh@hotmail.com

\section{A. S. Dharampal • P. J. de Feyter}

Department of Cardiology, Erasmus MC,

Rotterdam, the Netherlands

P. J. de Feyter

Department of Radiology, Erasmus MC, Room Hs 207,

's-Gravendijkwal 230, 3015 CE, Rotterdam, the Netherlands
Coronary calcification can be easily detected by computed tomography (CT). The epicardial vessels can be readily identified on a non-contrast enhanced CT scan (calcium scan), because the density of the coronary wall and blood is higher than the surrounding peri-coronary fat. Coronary calcium deposits have a 2 to 10 fold higher density than surrounding tissues and coronary calcium is identified as a hyper-attenuating coronary lesion above a threshold of 130 Hounsfield units (HU). The amount of calcium is quantified using the Agatston score (calcium score) which is derived from the product of the area of calcification $\left(\mathrm{mm}^{2}\right)$ and a factor determined by the maximal density (HU) within that area. The factor is $1,2,3,4$ with a density of 130 to 199,200 to 299,300 to 399 or $>400$ HU respectively [6].

The calcium scan does not require contrast medium and can be acquired with a low radiation dose of less than $1.0 \mathrm{mSv}$ [7]. This is associated with a lifetime attributable risk of cancer that for men and women at 50 years of age at 8 and 20 per 100,000 persons per year, respectively.

The calcium score is a significant predictor of risk of adverse cardiovascular events that has independent incremental value of risk prediction beyond the traditional risk prediction scores such as the Framingham risk score or the Euro risk score. Increasingly higher calcium scores are associated with increasingly higher risk of adverse cardiovascular events and all-cause mortality.

The calcium score can also be used to predict the presence of obstructive coronary artery disease (CAD).

\section{Calcium score to predict obstructive CAD}

The non-enhanced calcium scan represents an anatomic measure of the coronary plaque burden. The degree of a coronary obstruction is weakly correlated with the amount of calcium on a segment-by-segment basis, which is caused 
by the often occurring positive coronary wall remodelling at the site of the calcific plaque to preserve the coronary lumen size. Calcification is associated with both non-obstructive and obstructive coronary lesions and thus the calcium score is not specific for the presence of an obstructive lesion.

However, the total coronary calcium score is correlated with the presence of an obstructive angiographic coronary lesion [8].

A meta-analysis was performed from 16 studies of patients who were symptomatic and referred to invasive coronary angiography [8]. The sensitivity and specificity was $80 \%$ and $40 \%$ respectively (Table 1 ). The odds of calcium for detecting minimal CAD $(<50 \%)$ with no calcium present as reference was 6.8 (95\% CI 3.0-5.6), for $>50 \%$ obstruction $16.4(95 \%$ CI $5.1-53.1)$ and for $>70 \%$ obstruction 50 (95\% CI 24.1-103.0).

A positive calcium score moderately predicts the presence of significant coronary obstruction. Higher calcium scores, taking into account gender and age, are more predictive of obstructive CAD [9, 10]. This is illustrated in Figs. 1 and 2 which are taken from a study by Haberl et al. [9].

A calcium score of zero is associated with a very low prevalence of any coronary atherosclerotic plaque and is almost exclusively associated with the absence of an obstructive $(\geq 50 \%)$ lesion, although in young patients and in patients presenting with acute coronary syndrome a significant noncalcific plaque may be present, albeit that its presence is very low [11]. Patients with zero calcium have an excellent prognosis [11].

\section{Predictive value of calcium score in patients with moderate CKD}

Patients with end-stage chronic kidney disease (CKD) have (very) high coronary calcium scores and increased cardiovascular mortality which is mainly related to progressive

Table 1 Calcium score to predict $>50 \%$ coronary obstruction [8]

\begin{tabular}{ll}
\hline Subjects & 3683 \\
\hline Male \% & 67 \\
Mean age (years) & 55.3 \\
Prevalence calcium \% & 66 \\
Prevalence of significant CAD $\%$ & 57 \\
Sensitivity (weighted) \% & 80 range 68-100 \\
Specificity (weighted) \% & 40 range 21-100 \\
Predictive accuracy (weighted) \% & 59 range 41-95 \\
\hline
\end{tabular}

${ }^{a}$ significant $\mathrm{CAD}$ was defined as luminal irregularities in 2 reports, $>50 \%$ or $\geq 50 \%$ stenosis in 11 reports and $\geq 70 \%$ or $\geq 75 \%$ stenosis in 3 reports

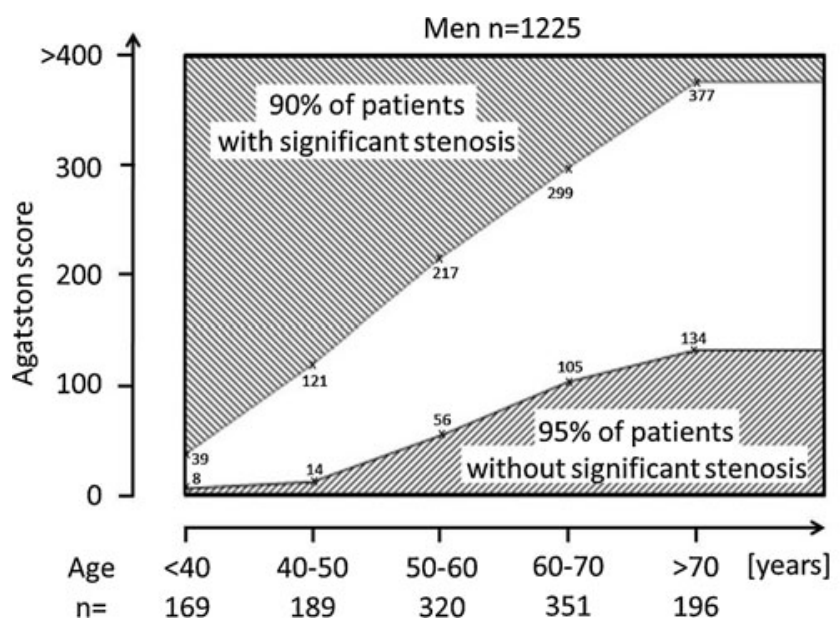

Fig. 1 Coronary calcium score prediction of the obstructive CAD in different age groups in men referred for coronary angiography. The lower scores define the thresholds for the $95 \%$ of patients without obstructive CAD. The higher scores represent the thresholds for the $90 \%$ of patients with obstructive CAD. The prediction is uncertain within the orange area. Adapted from Haberl et al. J Am Coll Cardiol $2001 ; 37: 451-7$

coronary atherosclerosis with associated higher calcium scores, but the presence of other risk factors, hypertension or diabetes, also play a role. But also patients in earlier stages of CKD have a higher prevalence of calcium score and CAD than patients without CKD [12, 13]. Reports about the predictive value of the coronary calcium score in patients with end-stage CKD to identify obstructive CAD are conflicting and studies in patients with moderate CKD are lacking [14-16]. In this issue of the journal Yiu et al. compared the value of the calcium score to predict obstructive CAD in patients with moderate CKD $(n=69)$ with patients without CKD $(n=635)$ [17]. They demonstrated that the calcium score was almost equally predictive in both patient groups, with the caveat that the optimal cut-off value of the score is

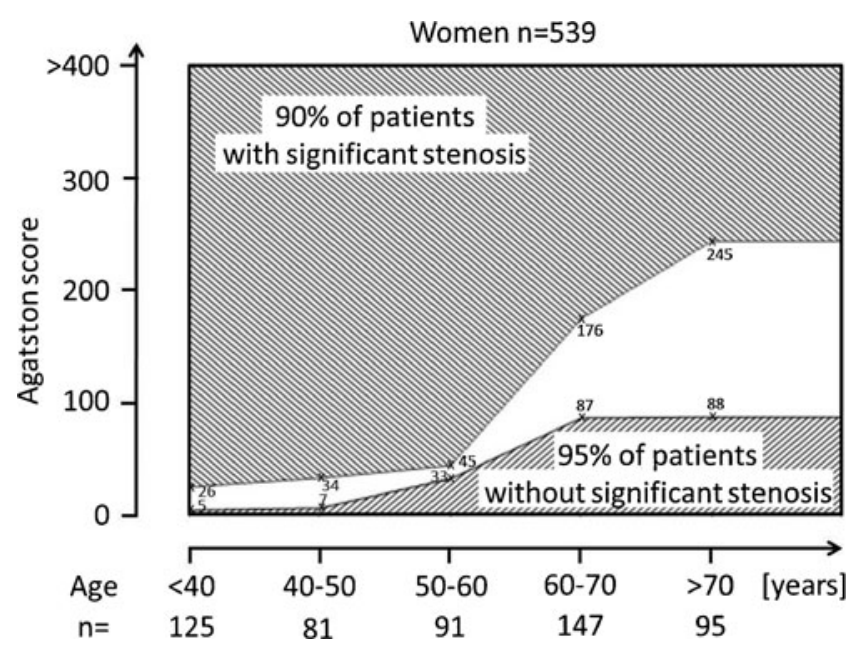

Fig. 2 Coronary calcium score prediction in women. See legend Fig. 1 
higher in patients with moderate CKD. This may be expected because in CKD patients coronary calcification not only takes place in the sub-intima (as is the case in coronary atherosclerosis) but in addition also in the media of the coronary wall (media-sclerosis or Moenckebergtype atherosclerosis) [18].

CT imaging cannot distinguish between subintimal calcification and medial calcification. Nevertheless, the authors demonstrated that the calcium score is moderately predictive to identify obstructive CAD in patients with moderate CKD with a sensitivity of $73 \%$ and a specificity of $70 \%$. The role of medial calcification as a marker of cardiovascular risk or as a predictor of obstructive CAD is not well defined due to the lack of an adequate imaging modality.

Coronary calcium scanning is appealing because it is more than a risk factor, it is the disease in itself. The question remains: should we use the coronary calcium score as a predictor of obstructive CAD in a general population or in this case in patients with moderate CKD? Arguments in favour of its use are the convenience, no use of contrast medium, low radiation exposure and low costs. A significant argument against is the low specificity and high false-positive rate of the calcium scan to predict obstructive CAD in the general population and the moderate sensitivity and specificity in patients with moderate CKD. One may argue that instead CT coronary angiography is performed to directly identify obstructive CAD which nowadays can be acquired with a very low radiation dose $(<1 \mathrm{mSv})$ and high diagnostic accuracy, although it is less convenient, requires the supervision of a radiologist, use of contrast medium, and is more costly.

In any case, further studies are needed to demonstrate that either a calcium scan or CT coronary angiography affects patients' management and improves prognosis.

\section{Funding None.}

\section{Conflict of interests None declared.}

Open Access This article is distributed under the terms of the Creative Commons Attribution License which permits any use, distribution, and reproduction in any medium, provided the original author(s) and the source are credited.

\section{References}

1. Johnson RC, Leopold JA, Loscalzo J. Vascular calcification: pathobiological mechanisms and clinical implications. Circ Res. 2006;99:1044-59.
2. Virmani R, Burke AP, Farb A, et al. Pathology of the vulnerable plaque. J Am Coll Cardiol. 2006;47:C13-8.

3. Rumberger JA, Simons DB, Fitzpatrick LA, et al. Coronary artery calcium area by electron-beam computed tomography and coronary atherosclerotic plaque area. A histopathologic correlative study. Circulation. 1995;92:2157-62.

4. Motoyama S, Kondo T, Sarai M, et al. Multislice computed tomographic characteristics of coronary lesions in acute coronary syndromes. J Am Coll Cardiol. 2007;50:319-26.

5. Kataoka Y, Wolski K, Uno K, et al. Spotty calcification as a marker of accelerated progression of coronary atherosclerosis: insights from serial intravascular ultrasound. J Am Coll Cardiol. 2012;59:1592-7.

6. Agatston AS, Janowitz WR, Hildner FJ, et al. Quantification of coronary artery calcium using ultrafast computed tomography. J Am Coll Cardiol. 1990;15:827-32.

7. Kim KP, Einstein AJ, Berrington de Gonzalez A. Coronary artery calcification screening: estimated radiation dose and cancer risk. Arch Intern Med. 2009;169:1188-94.

8. O'Rourke RA, Brundage BH, Froelicher VF, et al. American College of Cardiology/American Heart Association Expert Consensus document on electron-beam computed tomography for the diagnosis and prognosis of coronary artery disease. Circulation. 2000;102:126-40.

9. Haberl R, Becker A, Leber A, et al. Correlation of coronary calcification and angiographically documented stenoses in patients with suspected coronary artery disease: results of 1,764 patients. J Am Coll Cardiol. 2001;37:451-7.

10. Budoff MJ, Diamond GA, Raggi P, et al. Continuous probabilistic prediction of angiographically significant coronary artery disease using electron beam tomography. Circulation. 2002;105:1791-6.

11. Sarwar A, Shaw LJ, Shapiro MD, et al. Diagnostic and prognostic value of absence of coronary artery calcification. JACC Cardiovasc Imaging. 2009;2:675-88.

12. Cho I, Min HS, Chun EJ, et al. Coronary atherosclerosis detected by coronary $\mathrm{CT}$ angiography in asymptomatic subjects with early chronic kidney disease. Atherosclerosis. 2010;208:406-11.

13. Kestenbaum BR, Adeney KL, de Boer IH, et al. Incidence and progression of coronary calcification in chronic kidney disease: the Multi-Ethnic Study of Atherosclerosis. Kidney Int. 2009;76:9918.

14. Haydar AA, Hujairi NM, Covic AA, et al. Coronary artery calcification is related to coronary atherosclerosis in chronic renal disease patients: a study comparing EBCT-generated coronary artery calcium scores and coronary angiography. Nephrol Dial Transplant. 2004;19:2307-12.

15. Robinson J, Tan AU, Wilensky RL, et al. Electron-beam computerized tomography correlates with coronary angiogram in chronic kidney disease patients. Am J Nephrol. 2007;27:247-52.

16. Sharples EJ, Pereira D, Summers S, et al. Coronary artery calcification measured with electron-beam computerized tomography correlates poorly with coronary artery angiography in dialysis patients. Am J Kidney Dis. 2004;43:313-9.

17. Yiu KH, de Graaf FR, van Velzen JE, et al. Different value of coronary calcium score to predict obstructive coronary artery disease in patients with and without moderate chronic kidney disease. Neth Heart J. 2013. doi:10.1007/s12471-013-0409-8. Epub ahead of print

18. Stavroulopoulos A, Porter CJ, Pointon K, et al. Evolution of coronary artery calcification in patients with chronic kidney disease Stages 3 and 4, with and without diabetes. Nephrol Dial Transplant. 2011;26:2582-9. 